\title{
First case of intestinal leiomyosarcoma in a bearded dragon: ultrasonographic findings
}

\author{
ANNA ŁOJSZCZYK-SZCZEPANIAK, ANNA ŚMIECH*, NADIA CHLEBICKA**, \\ KLAUDIUSZ O. SZCZEPANIAK ${ }^{* * *}$, PAWEK KLIMIUK* \\ Laboratory of Radiology and Ultrasonography, Department and Clinic of Animal Surgery, \\ *Department of Pathological Anatomy, Faculty of Veterinary Medicine, \\ University of Life Sciences in Lublin, Głęboka 30, 20-612 Lublin, Poland \\ **Private veterinary practice "Rex", Balladyny 6, 20-601 Lublin, Poland

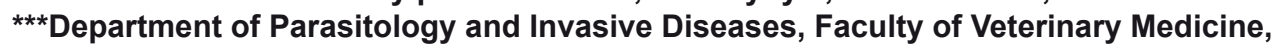 \\ University of Life Sciences in Lublin, Akademicka 12, 20-950 Lublin, Poland
}

Łojszczyk-Szczepaniak A., Śmiech A., Chlebicka N., Szczepaniak K. O., Klimiuk P. First case of intestinal leiomyosarcoma in a bearded dragon: ultrasonographic findings

Summary

The paper presents a case of leiomyosarcoma of the gastrointestinal tract in a bearded dragon (Pogona vitticeps) with an ultrasound and histopathological description of pathological changes. According to our knowledge, leiomyosarcoma of the gastrointestinal tract and its ultrasound features have not been reported in lizards yet.

A male bearded dragon (Pogona vitticeps), aged 4 years, was referred for radiological and ultrasound examination with a history of apathy and loss of appetite. A preliminary diagnosis of an advanced neoplasia process in the caudal coelomic cavity was established by diagnostic imaging methods. The owner decided to euthanize the animal and agreed to a post-mortem examination. After euthanasia, a histopathological examination was performed. In a radiological examination, an irregularly marginated radiopacity measuring $5.8 \times 4.0 \mathrm{~cm}$ was visible in the central part of the coelom. Serosal margin details in the coelomic cavity were decreased, and differentiation of coelomic organs was impossible. An ultrasound examination confirmed a large amount of free echogenic fluid in the coelomic cavity. The liver had increased echogenicity, and a hypoechoic focal lesion was found in the right lobe. Mixed echogenicity masses were visible in the caudal coelom adjacent to the small intestinal loops. In one of the adjacent intestinal loops, a focal thickening of the small intestinal wall was observed with loss of the normal layered architecture. The mass had complex and mostly low echogenicity with a cystic lesion present in the centre. Necropsy confirmed the presence of proliferative lesions in the body cavity, originating from the intestine and infiltrating the mesentery and the lumbar spine area as well as the gonads located at this site. On the basis of the histological structure of the intestinal tumour, the neoplastic proliferation was classified, according to the WHO classification, to a group of malignant tumours with characteristics of leiomyosarcoma and arising from the smooth muscles. The ultrasonographic features of gastrointestinal leiomyosarcoma in that lizard corresponded to features found in dogs, in which such tumours occur as eccentric, poorly echogenic masses with anechoic cavities in large changes. Leiomyosarcoma in reptiles can be locally invasive as well. Metastatic foci are also possible, especially in the liver.

Keywords: leiomyosarcoma, ultrasonography, gastrointestinal tract, bearded dragon

Leiomyosarcoma (LMS) is one of the most common sarcomas of the gastrointestinal (GI) tract (8). This type of tumour originates from the muscularis propria of the intestinal wall. It is characterised by slow growth and local invasion. Metastases, ranging from $16 \%$ to $37.5 \%$, are most frequently found in the jejunal lymph nodes, liver and peritoneum $(2,8)$.

It constitutes from $10 \%$ to $30 \%$ of reported intestinal tumours in dogs. LMS is reported less often in other species of animals $(2,8)$.
In the veterinary literature, there are only three reports of LMS in reptiles. Two of them concern this type of neoplasia in the gastrointestinal (GI) tract of snakes. The first report describes LMS of the small intestine in a Texas Indigo Snake (Dymarchon corais), and the second deals with LMS in the cloaca of an Egyptian Cobra (Naja naja) (11). One case of primary ovarian LMS in a bearded dragon (Pogona vitticeps) was observed by Sonntag et al. (12). According to our knowledge, LMS of the GI tract has not been 
reported in lizards or turtles. Furthermore, the ultrasonographic features of GI neoplasia in reptiles also remain unknown.

Here, we present a case of LMS of the GI tract in a bearded dragon (Pogona vitticeps) with an ultrasound and histopathological description of pathological changes.

\section{Material and methods}

A male lizard, bearded dragon, aged 4 years, was referred by a private clinic to the Laboratory of Radiology and Ultrasonography, Department and Clinic of Animal Surgery, for radiological examination. The animal was presented with a 3-week history of apathy and loss of appetite. In the clinical examination, a large solid mass was palpable in the caudal coelomic region. Radiographs were taken in dorsoventral and right lateral recumbency. Next, an ultrasound examination was performed by means of a Mindray DC-7 machine with a $12 \mathrm{MHz}$ linear probe. Based on the radiological and ultrasound examination, a preliminary diagnosis of advanced neoplasia process in the caudal coelomic cavity with possible metastasis to the liver was established. However, additional laboratory tests were recommended to determine neoplasia characteristics. Because of poor diagnosis and a high cost of further laboratory tests, the owner decided to euthanize the animal.

During necropsy, samples for histopathological examination were collected and fixed in 10\% formalin buffered to $\mathrm{pH}$ 7.2. Subsequently, tissue sections were dehydrated in increasing concentrations of alcohol solutions and acetone, immersed in xylene, and formed into paraffin blocks in a tissue processor. Tissue sections with a thickness of $4 \mu \mathrm{m}$ were prepared on a sliding microtome and placed on a microscope slide. Specimens for histopathological examination were stained with haematoxylin and eosin (HE stain) and observed under a light microscope.

\section{Results and discussion}

Diagnostic imaging findings. A survey radiograph showed a slight increase in lung opacity and obscured pulmonary details. The lung margins were displaced medially by homogeneous opacity, which was about $2 \mathrm{~cm}$ wide. This image corresponded to free fluid in body cavity. In the central part of the coelom, irregularly marginated radiopacity measuring $5.8 \times 4.0 \mathrm{~cm}$ was visible. A well-developed fat body was located ventrally and laterally to the radiopacity. Serosal margin details in the coelomic cavity were decreased and differentiation of coelomic organs was impossible.

The ultrasound examination confirmed a large amount of free echogenic fluid in the coelomic cavity. The liver had a granular structure with echogenicity similar to that of the caudally located fat bodies. A hypoechoic single focal lesion was found in the right lobe, near the gallbladder. The nodule measured $0.9 \times 0.8 \mathrm{~cm}$ and was smoothly marginated (Fig. 1) During the Doppler examination, the nodule had low flow mainly in its central and peripheral parts.

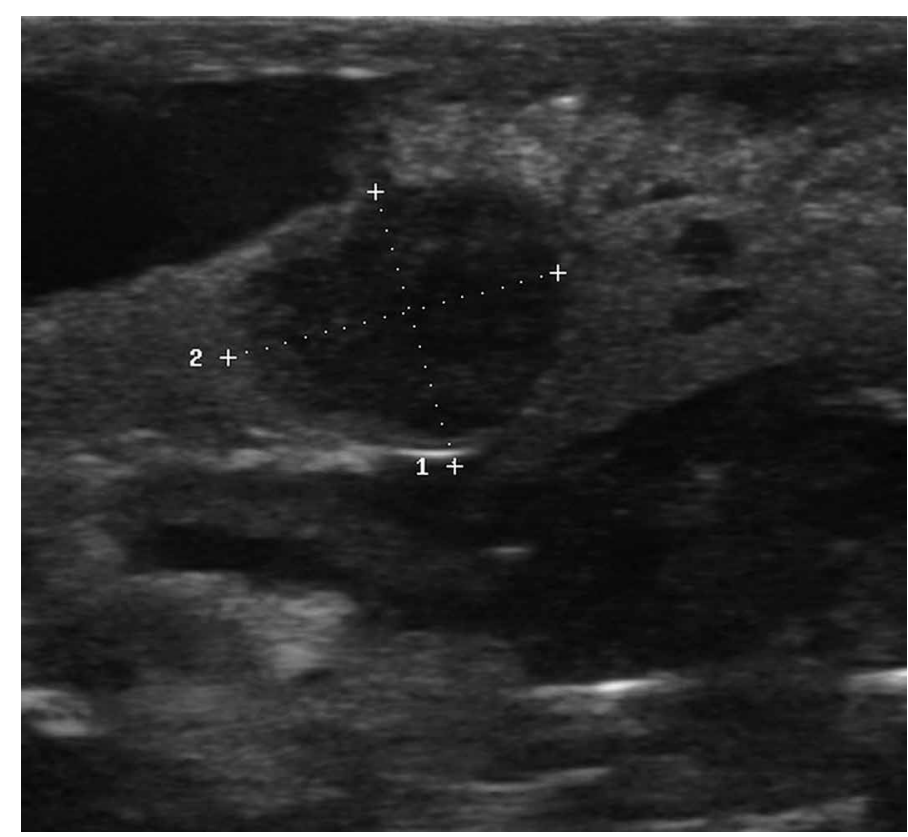

Fig. 1. Ultrasound findings. A hypoechoic nodule in the right lobe of the liver. The gallbladder is visible on the left

Subsequently, mixed echogenicity masses in the caudal coelom adjacent to the small intestinal loops were visible (Fig. 2). In one of the adjacent loops $(1.3 \mathrm{~cm}$ in length), a focal thickening (to $0.8 \mathrm{~cm}$ ) of the small intestinal wall was observed with loss of the normal layered architecture. The mass had complex and mostly low echogenicity. A cystic lesion measuring $0.3 \times 0.2 \mathrm{~cm}$ was present in the centre (Fig. 3 ).

Due to the presence of numerous proliferative changes and a large amount of fluid, the margins of

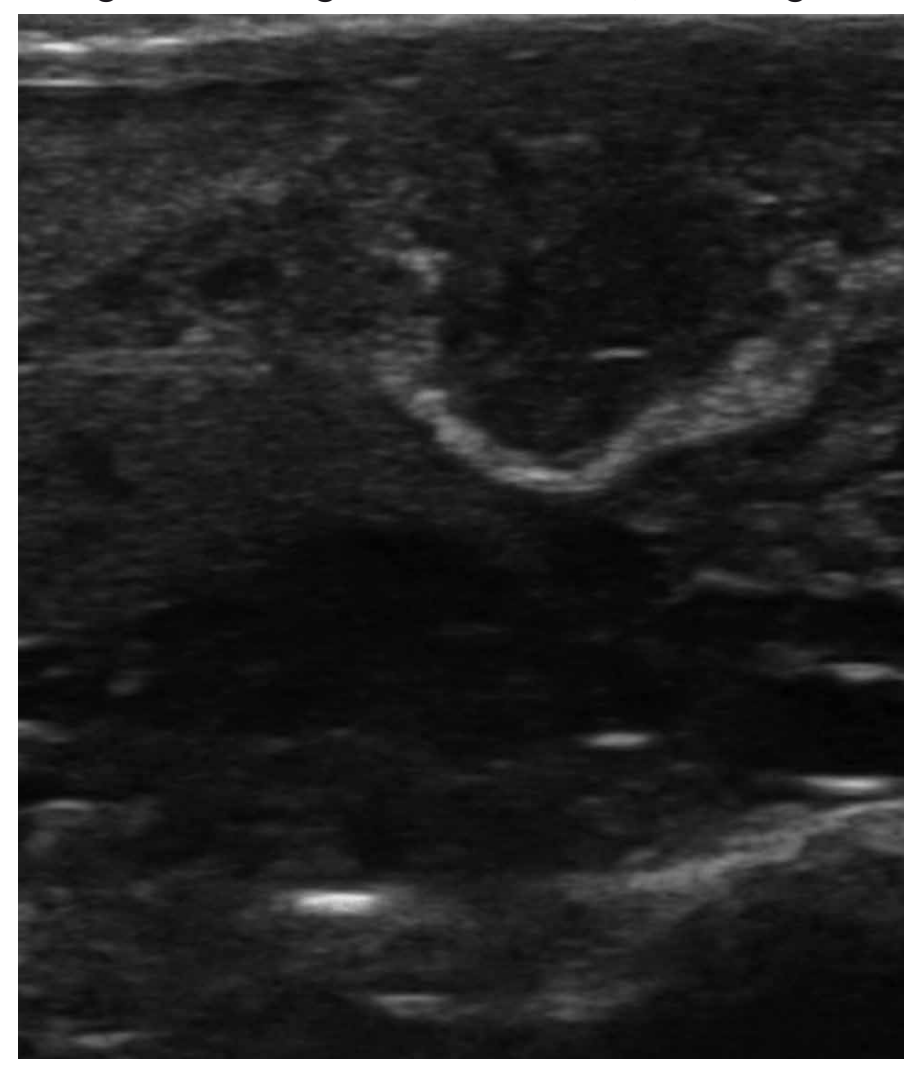

Fig. 2. Ultrasound findings. Mixed echogenicity masses found in the caudal coelom, adjacent to the small intestinal loops 


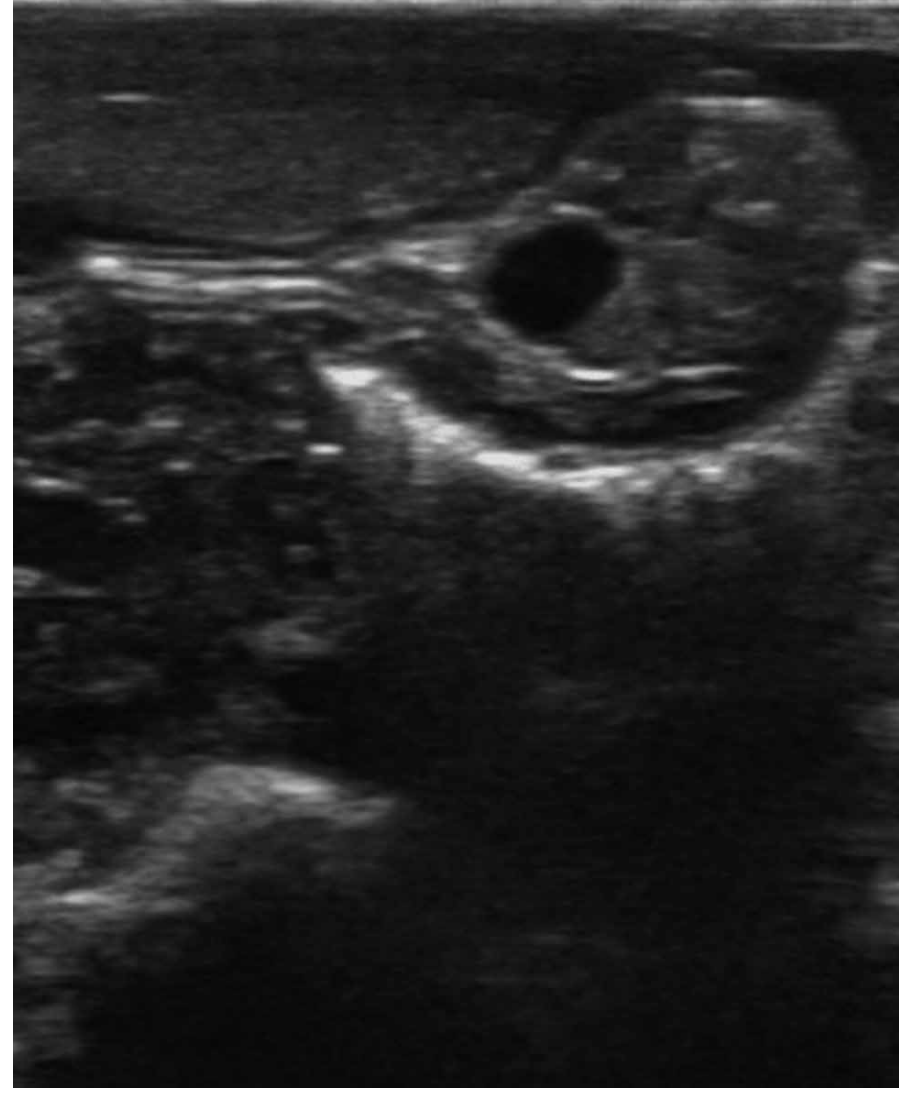

Fig. 3. Ultrasound findings. An inhomogeneous, mixed echogenicity intestinal mass with a cystic lesion in the centre. The loss of the normal layered architecture is visible

organs located in the caudal part of the coelomic cavity, including the intestines, were difficult to identify. In the other parts of the coelom, the small intestine had a normal ultrasonographic appearance. The intestinal wall was $0.05-0.12 \mathrm{~cm}$ wide, and the wall layering was normal.

Post-mortem examination findings. Necropsy confirmed the presence of proliferative lesions in the body cavity, originating from the intestine and infiltrating the mesentery and the lumbar spine area as well as the gonads located at this site. The necropsy also revealed distinct features of liver steatosis. In the right lobe of the liver there was a large nodule, well-demarcated and milky in colour, which corresponded to the lesion previously identified during the ultrasonographic examination. In the coelomic cavity, a large amount of bloody effusion was found, as well as adhesions between the liver and the peritoneum.

Histopathological findings. Histopathological examination of liver sections confirmed the presence of the fatty degeneration identified by ultrasound. The microscopic image showed areas of hepatocytes with numerous vacuoles in the cytoplasm, often causing the cell nucleus to be displaced to the edge or even to disappear. Between hepatic cells, clearly visible clusters of brown-black melanomacrophages were observed. The microscopic examination of the intestinal tumour revealed fusiform cancer cells with hyperchromatic cell nuclei proliferating from the intestinal smooth

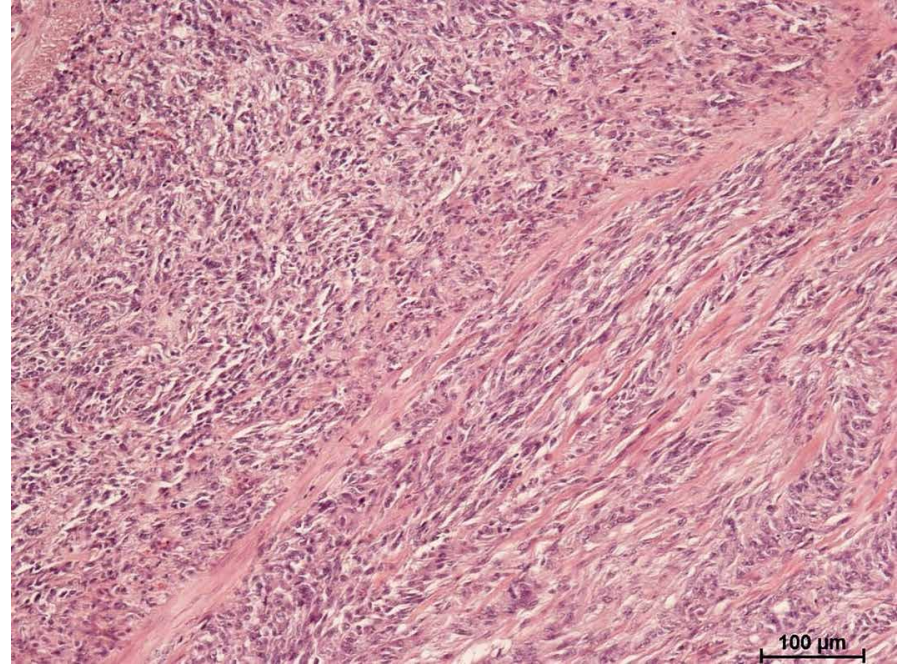

Fig. 4. Leiomyosarcoma, a mass from the intestinal wall in a bearded dragon. Magnif. $100 \times$

muscle. The tumour-forming cells, which were mostly chaotically and disorderly structured, infiltrated deeper layers of muscles (Fig. 4). These cells clearly exhibited anisocytosis, anisokaryosis and high mitotic activity. Between the layers of tumour cells, there were areas of necrosis and haemorrhage. On the basis of histological structure, the neoplastic proliferation was classified, according to the WHO classification, to a group of malignant tumours with characteristics of leiomyosarcoma and arising from the smooth muscles (4).

Descriptions of tumours of the GI tract in lizards are very rarely found in the scientific literature $(5-7,12)$. So far, according to the authors' knowledge, two research papers have been published, which confirm a case of colon carcinoma in a Komodo dragon (Varanus komodoensis) and gastric neuroendocrine carcinoma in a bearded dragon (Pogona vitticeps) $(6,7)$.

The ultrasound examination was of considerable importance in the course of diagnostic evaluation. A large amount of free fluid accumulated in the body cavity resulted in a loss of serosal details in the X-ray image, making the assessment of internal organs difficult. Moreover, the radiographic evaluation of the organs of the coelom is difficult even under normal conditions because of the anatomic characteristics of reptiles (11).

Ultrasonographic evaluation of the GI tract is based on the assessment of wall thickness, wall layer identification, wall symmetry, extent of the lesions, nature of the GI contents, motility and systemic involvement (8). Similar rules, with some restrictions, can be applied in reptiles (1). According to tests carried out by Bucy et al. on 14 healthy subjects, the proper thickness of the GI tract walls in bearded dragons amounts to $0.13 \pm 0.04$ for the stomach, $0.31 \pm 0.1$ for the small intestine and $0.11 \pm 0.03 \mathrm{~cm}$ for the colon. These data are consistent with stomach and intestine measurement results obtained in the present study. Apart from the intestinal segment with neoplastic proliferation, the GI 
tract wall did not show any thickening compared to the values reported by Bucy et al. During the examination of the lizard with a $12 \mathrm{MHz}$ probe, it was also possible to see alternating layers of the small intestine. It was not possible, however, to identify all layers, unlike in the case of companion animals. All layers of the small intestine observed were of the same width, unlike in dogs and cats, whose mucous membrane is the thickest and best separated layer (10). Proliferative lesions were observed only in a $1.2 \mathrm{~cm}$ section of the intestine, where the thickness of the intestinal wall was up to $0.8 \mathrm{~cm}$ with a loss of the normal layered architecture. A significant asymmetry of the nodule lesion growing centrifugally was also demonstrated. At the same time, neither a dilation of intestinal lumen, nor signs of improper peristalsis were identified, which could suggest a GI obstruction. The small intestine was empty because the animal had not eaten for a long time.

Because of the size and extent of the neoplastic process invading the surrounding tissues, further ultrasound evaluation was greatly impeded. Numerous proliferative lesions found during the ultrasound examination could not be associated with any internal organ. Only during the post-mortem examination it was found that the neoplastic process had infiltrated the mesentery. These observations may be consistent with the characteristics of leiomyosarcoma in other animal species in which the sarcoma is described as locally invasive (2).

Metastatic foci have been reported in animals with leiomyosarcoma (8). In the bearded dragon examined, the focal liver lesion was highly suggestive of the presence of a metastatic focus. In this case, however, there was no histopathological confirmation, because the nodule was not further analysed histopathologically. The liver tissue, which showed substantially increased echogenicity compared with the fat body during the ultrasound examination and presented distinct features of steatosis in the post-mortem examination, was examined histopathologically. Histopathological results confirmed the initial diagnosis.

It should also be noted that differences in anatomy and physiology between reptiles and companion animals can make the assessment and clinical relevance of hepatic lesions more difficult. Fatty liver may also be observed in reptiles under normal conditions during the breeding season because of the oestrogen stimulation of the liver to convert lipids from fat reserves into yolk. This conversion process is most intense in females before hibernation and least intense in males during late hibernation (3). Therefore, an increased echogenicity of the liver in reptiles should be evaluated carefully, especially in females. The diagnosis of steatosis should be considered together with data concerning medical history, with special attention paid to high-fat diet, decreased physical activity and clinical symptoms (3). In bearded dragons kept individually as exotic pets, hepatic lipidosis is commonly associated with infection and invasive diseases, such as adenovirosis and choleoeimeriosis (13). In the present case, however, lesions characteristic of infectious diseases were not observed either in ultrasound examination or in histopathologic analysis.

By analysing all data from the ultrasound examination, it can be concluded that the ultrasonographic features of gastrointestinal leiomyosarcoma in the lizard examined correspond to features found in dogs. In these animals, smooth muscle tumours appear mostly as eccentric, poorly echogenic masses with anechoic cavities in large changes $(8,9)$. A mechanical obstruction is very rare in such cases (9). During ultrasound examination, a focal thickening of the intestinal wall with loss of its layered architecture makes it possible to differentiate neoplastic processes from the inflammation of the GI tract $(8,9)$. It is likely that similar conclusions concerning reptiles could also be drawn if a larger number of cases were analysed. Although the layering of the walls in reptiles is not as easy to assess as it is in dogs and cats, the possibilities of GI tract assessment in reptiles may be enhanced by the use of higher frequency probes.

\section{References}

1. Bucy D. S., Guzman D. S. M., Zwingenberger A. L.: Ultrasonographic anatomy of bearded dragons (Pogonavitticeps). Vet. Radiol. Ultrasound. 2015, 8, 868-876 .

2. Cohen M., Post G. S., Wright J. C.: Gastrointestinal leiomyosarcoma in 14 dogs. J. Vet. Intern. Med. 2003, 17, 707-110.

3. Divers S. J., Cooper J. E.: Reptile Hepatic Lipidosis. Seminars in Avian and Exotic Pet Medicine. 2000, 3, 153-164.

4.Head K. W., Cullen J. M., Dubielzieg R. R., Misdorp W., Patnaik A. K., Tateyama S., van de Gaag I.: Histological Classification of Tumors of the Alimentary System of Domestic Animals, [in:] WHO International Histological Classification of Tumors of Domestic Animals. Second Series, Volume X. Armed Forces Institute of Pathology, Washington, D.C. 2003, p. 100-110.

5. Hernandez-Divers S. M., Garber M. M.: Neoplasia of reptiles with emphasis on lizards. Vet. Clin. North. Am. Exot. Anim. Pract. 2003, 1, 251-273.

6.Lyons J. A., Newman S. J., Greenacre C. B., Dunlap J.: A gastric neuroendocrine carcinoma expressing somatostatin in a bearded dragon (Pogona vitticeps). J. Vet. Diagn. Invest. 2010, 22, 316-320.

7. Mauldin N. G., Done L. B.: Oncology, [in:] Mader D. (ed.): Reptile Medicine and Surgery. Elsevier, St. Louis, Missouri 2006, p. 299-321.

8. Myers III N. C., Pennick D. G.: Ultrasonographic diagnosis of gastrointestinal smooth muscle tumors in the dog. Vet. Radiol. Ultrasound. 1994, 5, 391-397.

9. Pennick D., Smyers B., Webster C., Rand W., Moore A. S.: Diagnostic value of ultrasonography in differentiating enteritis from intestinal neoplasia in dogs. Vet. Rad. Ultrasound 2003, 5, 570-575.

10. Pennick D. G., Nyland T., Kerr L. Y., Fisher P. E.: Ultrasonographic evaluation of gastrointestinal diseases in small animals. Vet. Radiol. Ultrasound. 1990, 3, 134-141.

11. Silverman S.: Diagnostic Imaging, [in:] Mader D. (ed.): Reptile Medicine and Surgery. Elsevier, St. Louis, Missouri 2006, p. 471-489.

12. Sonntag F., Schroff C., Dietz J., Heckers K.: Metastasierendes Leiomyosarkom des Ovars bei einer Bartagame (Pogona vitticeps) - ein Fallbericht. Prakt. Tierarzt 2014, 95, 518-523.

13. Szczepaniak K. O., Tomczuk K., Łojszczyk-Szczepaniak A., Łopuszyński W.: Reclassification of Eimeria pogonae Walden (2009) as Choleoeimeria pogonae comb. nov. (Apicomplexa: Eimeridae). Parasitol. Res. 2015, DOI 10.1007/ s00436-015-4787-2.

Corresponding author: Dr. Anna Lojszczyk-Szczepaniak PhD, ul. Głęboka 30, 20-612 Lublin, Poland; e-mail: anna.lojszczyk@gmail.com 